\title{
To confirm the existence of Black hole cosmology
}

\author{
U. V.S. Seshavatharam ${ }^{1 *}$ and S. Lakshminarayana ${ }^{2}$ \\ ${ }^{1}$ Honorary faculty, I-SERVE, Alakapuri, Hyderabad-35, AP, India \\ ${ }^{2}$ Dept. of Nuclear Physics, Andhra University, Visakhapatnam-03, AP, India \\ *Corresponding author E-mail: seshavatharam.uvs@gmail.com
}

\begin{abstract}
It may be noted that, increased redshifts and increased distances forced Edwin Hubble to propose the Hubble's law. In fact there is no chance or scope or place for 'galaxy receding'. If it is possible to show that, (from the observer) older galaxy's distance increases with its 'age', then automatically the concepts 'galaxy receding' and 'accelerating universe' can be put for a revision at fundamental level. Its only our belief in its 'given' (Doppler shift based) unproven cosmological interpretation. Even then, merely by estimating galaxy distance and without measuring galaxy receding speed, one cannot verify its acceleration. Clearly speaking: two mistakes are happening here. 1) Assumed galaxy receding speed is not being measured and not being confirmed. 2) Without measuring and confirming the galaxy receding speed, how can one say and confirm that it (galaxy) is accelerating. It is really speculative and unfortunate also. During cosmic expansion, assuming past and present galaxies (which actually found to have gigantic structures) as 'points' and guessing photons coming from that galactic point particles seem to be ad-hoc. If light is coming from the atomic matter of the gigantic galaxy, then cosmic redshift can be interpreted as an index of the galactic atomic 'light emission mechanism'. In no way it seems to be connected with 'galaxy receding'. Whatever may be the expression, definitions of cosmic red shift seem to be ad-hoc and not absolute. Hence with redshift concept - one may not be able to understand the actual rate of cosmic expansion and actual cosmic geometry. Some cosmologists use the term 'Hubble volume' to refer to the volume of the observable universe. At any given time, the product of 'critical density' and 'Hubble volume' gives a characteristic cosmic mass and it can be called as the 'Hubble mass'. Interesting thing is that, Schwarzschild radius of the Hubble mass again matches with the Hubble length. Most of the cosmologists believe that this is merely a coincidence. If one is able to show its applications in different areas of fundamental physics, certainly it can be given more significance and superiority compared to the mysterious 'dark energy'. At any given cosmic time, 'Hubble length' can be considered as the gravitational or electromagnetic interaction range. Hubble volume and Hubble mass play a crucial role in quantum physics, nuclear physics, atomic physics and particle physics. If universe constitutes so many galaxies, if each galaxy constitutes a central fast growing and (light speed) spinning black hole and if black hole geometry is more intrinsic than its 'mass' and 'mass density', then considering universe as a 'growing and light speed rotating black hole' may not be far away from reality. By increasing the number of applications of Hubble mass and Hubble volume in other areas of fundamental physics, slowly and gradually and in a progressive way concepts of Black hole Cosmology can be strengthened and can also be confirmed. Unknowingly the fundamental physical laws are being developed, being executed and being proven inside and under the background of a growing and light speed rotating black hole universe. It can be suggested that, 'quantum of angular momentum' may be due to the cosmological manifestation and 'discrete nature' of angular momentum may be due the discrete nuclear matter. In any bound system, 'operating force' only plays major role in maintaining the 'existence of the bound system' and 'angular momentum' is one of the result. If one is able to make the operating force as discrete, then automatically one can observe a discrete structure like discrete radii, discrete angular momentum and discrete energy levels. Alternatively if atomic nucleus constitutes any fixed number of protons and any fixed number of neutrons, it is possible to guess that- nuclear mass is discrete. If nuclear matter is discrete, it is also possible to have a discrete atomic structure . In this new direction authors noticed some interesting and very strange coincidences. Main problem is that, the new relations are very simple to understand and very difficult to confirm. The most important point to be noted here is that, synthesis of elementary physical constants seem to be more fundamental than the 'cosmological nucleosynthesis'.
\end{abstract}

Keywords: Hubble Length, Hubble Volume, Mach's Principle, Hubble mass, Light speed rotating black hole, Hydrogen atom, Quantum of angular momentum, Uncertainty relation, Redshift, Coulomb mass, CMBR energy density. 


\section{Introduction}

In physics history, for any new idea or observation or new model - at the very beginning - their existence was very doubtful. The best examples were : 1) Existence of atom 2) Existence of quantum of energy 3) Existence of integral nature of angular momentum 4) Existence of wave mechanics 5) Six quarks having fractional charge 6) Confirming the existence of muon/pion 7) Existence of Black holes 8) Black hole radiation 9) Einstein's cosmological Lambda term 10) Cosmic red shift 11) Discovery of CMBR 12) Accelerating universe and Dark energy [1-11]. The evidence for dark energy is only indirect and many things about the nature of dark energy remain matters of speculation. Not only that, in understanding the basic concepts of unification or TOE, role of dark energy and dark matter is insignificant.

Professor Peter Rowland says [12]- what is often described as the 'standard model' of cosmology is much less certain than the standard model of particle physics, much less supported by experimental evidence and much less coherent. A number of ad hoc 'fixes' are introduced to incorporate such phenomena as the large scale flatness of space-time, the rotation curves of galaxies, and the apparent acceleration in the expansion of the universe. The whole theory would be unsustainable if a non-expansionary explanation of the cosmological redshift should emerge. Santilli's prediction and observation of the isoredshift [13] from a physical theory with applications beyond the point particles and waves in vacuum to which Einstein's relativity theory is restricted requires us to look again at the possibility that other versions of cosmology may be more coherent and that theories of the physical phenomena involved (like the author's own) can be developed independently of any particular cosmology at all.

\subsection{Major shortcomings of modern cosmology}

A) It may be noted that, increased redshifts and increased distances forced Edwin Hubble to propose the Hubble's law. In fact there is no chance or scope or place for 'galaxy receding'. Its only our belief in its 'given' (Doppler shift based) interpretation. Even then, merely by estimating galaxy distance and without measuring galaxy receding speed, one cannot verify its acceleration. Clearly speaking: two mistakes are happening here. 1) Assumed galaxy receding speed is not being measured and not being confirmed. 2) Without measuring and confirming the galaxy receding speed, how can one say and confirm that it (galaxy) is accelerating. It is really speculative and unfortunate also.

B) During cosmic expansion, assuming past and present galaxies (which actually found to have gigantic structures) as 'points' and guessing photons coming from that galactic point particles seem to be ad-hoc. If light is coming from the atomic matter of the gigantic galaxy, then redshift can be interpreted as an index of the galactic atomic 'light emission mechanism'. In no way it seems to be connected with 'galaxy receding' [3,14,15].

C) If cosmic expansion is continuous and accelerating and redshift is a measure of cosmic expansion, then 'rate of increase in redshift' can be considered as a measure of cosmic 'rate of expansion'. Then there is no possibility to observe a 'constant' red shift [16,17]. More over the current definition of red shift seems to be ad-hoc and not absolute hence one may not be able to understand or confirm the actual cosmic rate of expansion.

D) According to the modern cosmological approach, bound systems like 'atoms' which found to be the major constituents of galactic matter - will not expand with cosmic expansion/acceleration. As per the present observational data this may be true. It might be the result of ending stage of expansion also. In this regard, without considering and without analysing the past data, one can not come to a conclusion. If it is not possible to collect the past data, theoretically it may be possible to proceed further in this new direction.

E) Even though it was having strong footing, Mach's principle was not implemented successfully.

F) Even though the whole physics strictly follow the 'constancy of speed of light', cosmic acceleration seems to violate it. This is really doubtful.

G) There is no scientific evidence for the Friedmann's second assumption. We believe it only on the grounds of modesty [18].

H) Drop in 'cosmic temperature' can be considered as a measure of cosmic expansion and 'rate of decrease in cosmic temperature' can be considered as a measure of cosmic 'rate of expansion'. But if rate of decrease in temperature is very small and is beyond the scope of current experimental verification, then the two possible states are: a) cosmic temperature is decreasing at a very slow rate and universe is expanding at a very slow rate and b) there is no 'observable' thermal expansion and there is no 'observable' cosmic expansion.

I) If 'Dark energy' is the major outcome of the 'accelerating universe', it is very important to note that - in understanding the basic concepts of unification or other fundamental areas of physics, role of dark energy is very insignificant. So far no ground based experiment confirmed the existence of dark energy. There is no single clue or definition or evidence to any of the natural physical properties of (the assumed) dark energy.

J) Dimensionally it is perfectly possible to show that, the dimensions of Hubble's constant and angular velocity are same. If so considering Hubble's constant merely as an expansion parameter may not be correct. Please see the following sub-section 1.2. 
With these major short comings, if one feels, modern cosmology - a 'standard cosmology' - it is really illogical, speculative and unfortunate. If one is willing to think in this new direction, certainly other hidden things can also be surfaced out.

\subsection{The cosmic 'critical density' and its dimensional analysis and the cosmic rotation}

Recent findings from the University of Michigan suggest that the shape of the Big Bang might be more complicated than previously thought, and that the early universe spun on an axis. A left-handed and right-handed imprint on the sky as reportedly revealed by galaxy rotation would imply the universe was rotating from the very beginning and retained an overwhelmingly strong angular momentum [19]. An anonymous referee who reviewed the paper for Physics Letters said, "In the paper the author claims that there is a preferred handedness of spiral galaxies indicating a preferred direction in the universe. Such a claim, if proven true, would have a profound impact on cosmology and would very likely result in a Nobel prize". Galaxies spin, stars spin, and planets spin. So, why not the whole universe? The consequences of a spinning universe seem to be profound [19-33], natural and 'cosmic collapse' can be prevented. Thus 'cosmic (light speed) rotation' can be considered as an alternative to the famous 'repulsive gravity' concept.

With a simple derivation it is possible to show that, Hubble's constant $\left(H_{t}\right)$ represents cosmological angular velocity. Assume that, a planet of mass $(\mathrm{M})$ and radius $(\mathrm{R})$ rotates with angular velocity $\left(\omega_{e}\right)$ and linear velocity $\left(v_{e}\right)$ in such a way that, free or loosely bound particle of mass (m) lying on its equator gains a kinetic energy equal to potential energy as,

$$
\begin{aligned}
& \frac{1}{2} m v_{e}^{2}=\frac{G M m}{R} \\
& R \omega_{e}=v_{e}=\sqrt{\frac{2 G M}{R}} \text { and } \omega_{e}=\frac{v_{e}}{R}=\sqrt{\frac{2 G M}{R^{3}}}
\end{aligned}
$$

i.e Linear velocity of planet's rotation is equal to free particle's escape velocity. Without any external power or energy, test particle gains escape velocity by virtue of planet's rotation. Using this idea, 'Black hole radiation' and 'origin of cosmic rays' can be understood. Note that if Earth completes one rotation in one hour then free particles lying on the equator will get escape velocity. Now writing $M=\frac{4 \pi}{3} R^{3} \rho_{e}$,

$$
\begin{aligned}
& \omega_{e}=\frac{v_{e}}{R}=\sqrt{\frac{8 \pi G \rho_{e}}{3}} \text { Or } \omega_{e}^{2}=\frac{8 \pi G \rho_{e}}{3} \\
& \text { Density, } \rho_{\mathrm{e}}=\frac{3 \omega_{\mathrm{e}}^{2}}{8 \pi \mathrm{G}}
\end{aligned}
$$

In real time, this obtained density may or may not be equal to the actual density. But the ratio, $\frac{8 \pi G \rho_{\text {real }}}{3 \omega_{\text {real }}^{2}}$ may have some physical meaning. The most important point to be noted here, is that, as far as dimensions and units are considered, from equation (4), it is very clear that, proportionality constant being $\frac{3}{8 \pi G}$,

$$
\text { density } \propto(\text { angular velocity })^{2}
$$

Equation (4) is similar to "flat model concept" of cosmic "critical density"

$$
\rho_{c}=\frac{3 H_{t}^{2}}{8 \pi G}
$$

Comparing equations (4) and (6) dimensionally and conceptually, i.e.

$$
\begin{aligned}
& \rho_{e}=\frac{3 \omega_{e}^{2}}{8 \pi G} \text { with } \rho_{\mathrm{c}}=\frac{3 H_{\mathrm{t}}^{2}}{8 \pi \mathrm{G}} \\
& H_{t}^{2} \rightarrow \omega_{\mathrm{e}}^{2} \text { and } H_{t} \rightarrow \omega_{e}
\end{aligned}
$$

It is very clear that, dimensions of 'Hubble's constant' must be 'radian/second'. In any physical system under study, for any one 'simple physical parameter' there will not be two different units and there will not be two different physical 
meanings. This is a simple clue and brings "cosmic rotation" into picture. This is possible in a closed universe only. Cosmic models that depends on this "critical density" may consider 'angular velocity of the universe' in the place of 'Hubble's constant'. In the sense, 'cosmic rotation' can be included in the existing models of cosmology. Then the term 'critical density' appears to be the 'volume density' of the closed and expanding universe.

\subsection{Einstein's views on unification of gravity and electromagnetism}

Note that, Einstein, more than any other physicist, untroubled by either quantum uncertainty or classical complexity, believed in the possibility of a complete, perhaps final, theory of everything. [18]. He also believed that the fundamental laws and principles that would embody such a theory would be simple, powerful and beautiful. Physicists are an ambitious lot, but Einstein was the most ambitious of all. His demands of a fundamental theory were extremely strong. If a theory contained any arbitrary features or undetermined parameters then it was deficient, and the deficiency pointed the way to a deeper and more profound and more predictive theory. There should be no free parameters - no arbitrariness. According to his philosophy, electromagnetism must be unified with general relativity, so that one could not simply imagine that it did not exist. Furthermore, the existence of matter, the mass and the charge of the electron and the proton (the only elementary particles recognized back in the 1920s), were arbitrary features. One of the main goals of a unified theory should be to explain the existence and calculate the properties of matter.

\subsection{Classical limits of Force and Power}

It is accepted that a complete theory of the evolution of the universe will not be possible until the development of a successful quantum theory of gravity. The outstanding problem in particle physics today is the inclusion of gravity in a single, unified quantum theory of all the fundamental interactions. Particle physicists have long suggested that the four fundamental forces of nature (viz. the gravitational, electromagnetic, weak nuclear and strong nuclear forces) are separate, low energy manifestations of what was once a single force at times close to the Big Bang. It is postulated that as the universe expanded and cooled, this single force gradually broke down into the four separate interactions observed today. A detailed quantum theory that describes the electromagnetic and weak nuclear forces in terms of a single force (the electroweak interaction) was developed in the 1970's and dramatically verified by high-energy experiments in particle accelerators a decade later. More ambitious quantum theories that incorporate the strong nuclear force (Grand Unified Theories) have been developed, and some experimental support for these models has emerged. However, unification theories that seek to unify the force of gravity with all the other forces (Theories of Everything) remain elusive, as the gravitational interaction lacks a quantum formulation.

To unify cosmology, quantum mechanics and the four observed fundamental cosmological interactions - certainly a 'unified force' is required. In this connection $\left(c^{4} / G\right)$ can be considered as the classical force limit [16,17]. Similarly $\left(c^{5} / G\right)$ can be considered as the classical power limit. If it is true that $c$ and $G$ are fundamental physical constants in physics, then $\left(c^{4} / G\right)$ and $\left(c^{5} / G\right)$ can also be considered as fundamental compound physical constants. These limits are more powerful than the Uncertainty limit.

Simple applications of $\left(c^{4} / G\right)$ can be stated as follows.

1. Magnitude of force of attraction or repulsion between any two charged particles never crosses $\left(c^{4} / G\right)$.

2. Magnitude of gravitational force of attraction between any two massive bodies never crosses $\left(c^{4} / G\right)$.

3. Magnitude of mechanical force on a revolving/rotating body never crosses $\left(c^{4} / G\right)$.

4. Magnitude of electromagnetic force on a revolving body never crosses $\left(c^{4} / G\right)$.

Simple applications of $\left(c^{5} / G\right)$ can be stated as follows.

1. Mechanical power (either input or output) never crosses $\left(c^{5} / G\right)$

2. Electromagnetic power (either input or output) never crosses $\left(c^{5} / G\right)$

3. Thermal radiation power (either input or output) never crosses $\left(c^{5} / G\right)$

4. Gravitational radiation power (either input or output) never crosses $\left(c^{5} / G\right)$ 


\subsection{Current status of the Mach's principle and Hubble volume}

In theoretical physics, particularly in discussions of gravitation theories, Mach's principle [34] is the name given by Einstein to an interesting hypothesis often credited to the physicist and philosopher Ernst Mach. The idea is that the local motion of a rotating reference frame is determined by the large scale distribution of matter. There are a number of rival formulations of the principle. A very general statement of Mach's principle is 'local physical laws are determined by the large-scale structure of the universe'. This concept was a guiding factor in Einstein's development of the general theory of relativity. Einstein realized that the overall distribution of matter would determine the metric tensor, which tells the observer which frame is rotationally stationary.

One of the main motivations behind formulating the general theory of relativity was to provide a mathematical description to the Mach's principle. However, soon after its formulation, it was realized that the theory does not follow Mach's principle. As the theoretical predictions were matching with the observations, Einstein believed that the theory was correct and did not make any farther attempt to reformulate the theory to explain Mach's principle. Later on, several attempts were made by different researchers to formulate the theory of gravity based on Mach's principle. However most of these theories remain unsuccessful to explain different physical phenomena. In the standard cosmology, 'Hubble volume' or 'Hubble sphere' is a spherical region of the Universe surrounding an observer beyond which objects recede from that observer at a rate greater than the speed of light due to the expansion of the Universe. The commoving radius of a Hubble sphere (known as the Hubble radius or the Hubble length) is, $\left(c / H_{0}\right)$, where $(c)$ is the speed of light and $\left(H_{0}\right)$ is the Hubble constant. More generally, the term 'Hubble volume' can be applied to any region of space with a volume of the order of $(4 \pi / 3)\left(c / H_{0}\right)^{3}$. 'Hubble volume' can be considered as a key tool in cosmology and unification. Some cosmologists use the term 'Hubble volume' to refer to the volume of the observable universe. With reference to the Mach's principle and the Hubble volume, at any cosmic time, if 'Hubble mass' is the product of cosmic 'critical density' and the 'Hubble volume', then it can be suggested that, 1) Each and every point in the free space is influenced by the Hubble mass, 2) Hubble volume and Hubble mass play a vital role in understanding the properties of electromagnetic and nuclear interactions and 3) Hubble volume and Hubble mass play a key role in understanding the geometry of the universe. Thus in this paper an attempt is made to understand the basic unified concepts of 'electromagnetism', 'gravity' and 'strong interaction range' via the Hubble length, Hubble volume and Hubble mass [35,36].

The basic idea of unification is - 1) To minimize the number of physical constants and 2) To merge a group of different fundamental constants into one compound physical constant with appropriate interpretation. In this journey, the first step is to see the numerical coincidences, second step is to interpret the numerical coincidences and the third step is to synchronize the current interpretations and new interpretations. When the new interpretation disagrees with the current interpretation, generally with the help of emerging science and technology, discrepancies can be resolved with future observations and experiments. Mean while mathematical physics play a key role in understanding and analyzing the new and old interpretations. When the subject under consideration is very sensitive to human thoughts, observations and interpretations and when the subject under consideration is also related with large numbers, proposed accurate numerical coincidences and new interpretations may be given some consideration.

\subsection{To study the nucleus, atom and the universe in a combined manner}

The study of cosmology, its progress and evolution is yet to be properly made to arrive at reliable and convincing conclusions. The present study is a major step forward in this direction. Even though there were a number papers/books published on cosmology, the attempt for a comprehensive study on this subject, coupled with comparative studies with the modern cosmology on one hand and with the modern atomic physics on the other, was not made by anybody so far. Thus, the present study can be termed as a 'basic project' in this field. Cosmological observations through ground telescope or satellite telescope is a normal practice. In this paper under consideration, current cosmological changes can be understood by studying the atom and atomic nucleus through ground based experiments. It is an interesting part of the study of cosmology and fundamental interactions. So far no national or international university or institute has taken this subject for R\&D. This is quite unique and the openness in the subjects of cosmology and fundamental interactions can be eliminated. It is a challenging idea and 100 years of atomic, nuclear and cosmic physics can be refined and unified. Based on the proposed relations and applications, Hubble volume or Hubble mass, can be considered as a key tool in unification as well as cosmology. If it is possible to identify the atomic cosmological physical variable, then by observing the rate of change in its magnitude (on the cosmological time scale), the cosmic acceleration can be verified and thus the cosmic geometry can be confirmed from atomic and nuclear physics! Without the advancement of nanotechnology or femto-technology this may not be possible. Not only that, independent of the cosmic red shift and CMBR observations cosmic acceleration can be checked in this new direction. 


\section{Semi empirical applications of Mach's principle, Hubble volume and Hubble mass}

Note that till today quantitatively Mach's principle was not implemented successfully in cosmic and nuclear physics. If we do not yet know whether the universe is spatially closed or open, then the idea of 'Hubble volume' can be used as a tool in cosmology and unification. It seems to be a quantitative description to the Mach's principle. In the universe, if the critical density is $\rho_{c} \cong\left(3 H_{0}^{2} / 8 \pi G\right)$ and the characteristic Hubble radius is $R_{0} \cong\left(c / H_{0}\right)$, mass of the cosmic Hubble volume is $M_{0} \cong \frac{c^{3}}{2 G H_{0}}$ and its Swarzschild radius matches with $R_{0} \cong\left(c / H_{0}\right)$.

Most of the cosmologists believe that this is merely coincidence. If one is able to show its applications in different areas of fundamental physics, certainly it can be given more significance and superiority compared to the mysterious 'dark energy'. For the time being let us call this mass as 'Hubble mass'. With this definition, apart from cosmology, Mach's principle can be given a fundamental unified significance in quantum physics, atomic physics, nuclear physics and particle physics! Here, as a point of curiosity, if one is willing to consider this mass as a characteristic mass of the universe, very easily, Planck scale, cosmology and particle physics can be studied in a unified manner.

\subsection{Strange coincidences in unified atomic, nuclear and cosmic physics}

Professor Recami says [37]: Let us recall that Riemann, as well as Clifford and later Einstein, believed that the fundamental particles of matter were the perceptible evidence of a strong local space curvature. A theory which stresses the role of space (or, rather, space-time) curvature already does exist for our whole cosmos: General Relativity, based on Einstein gravitational field equations; which are probably the most important equations of classical physical theories, together with Maxwell's electromagnetic field equations. Whilst much effort has already been made to generalize Maxwell equations, passing for example from the electromagnetic field to Yang-Mills fields (so that almost all modern gauge theories are modeled on Maxwell equations), on the contrary Einstein equations have never been applied to domains different from the gravitational one. Even if they, as any differential equations, do not contain any inbuilt fundamental length: so that they can be used a priori to describe cosmoses of any size. Our first purpose is now to explore how far it is possible to apply successfully the methods of general relativity (GR), besides to the world of gravitational interactions, also to the domain of the so-called nuclear, or strong, interactions: namely, to the world of the elementary particles called hadrons. A second purpose is linked to the fact that the standard theory (QCD) of strong interactions has not yet fully explained why the hadron constituents (quarks) seem to be permanently confined in the interior of those particles; in the sense that nobody has seen up to now an isolated "free" quark, outside a hadron. So that, to explain that confinement, it has been necessary to invoke phenomenological models, such as the so-called "bag" models, in their MIT and SLAC versions for instance. The "confinement" could be explained, on the contrary, in a natural way and on the basis of a well-grounded theory like GR, if we associated with each hadron (proton, neutron, pion,...) a particular "cosmological model".

If $m_{p}$ is the rest mass of proton and $m_{e}$ is the rest mass of electron, it is noticed that,

$R_{s} \cong \frac{G \sqrt{M_{0} \sqrt{m_{p} m_{e}}}}{c^{2}} \cong\left(\frac{m_{p}}{m_{e}}\right)^{\frac{1}{4}}\left(\frac{M_{0}}{m_{e}}\right)^{\frac{1}{2}} \frac{G m_{e}}{c^{2}} \cong(1.37$ to 1.39$) \times 10^{-15} \mathrm{~m} \cong(1.37$ to 1.39$) \mathrm{fm}$

where $H_{0} \cong 70.4_{-1.4}^{+1.3} \mathrm{~km} / \mathrm{sec} / \mathrm{Mpc}$ [38-42] and $M_{0} \cong\left(c^{3} / 2 G H_{0}\right)$. In reality, this length is close to the observed strong interaction range or the characteristic nuclear unit radius [43-45]. Now the fundamental question to be answered is - is the characteristic nuclear size increases with cosmic time? Answer seems to be 'yes' and to be confirmed by the future science and technology. In terms of the proposed classical force limit, above expression can be expressed as $R_{s} \cong\left[\left(\frac{m_{e}}{m_{p}}\right)^{\frac{1}{4}}\left(\frac{m_{e}}{M_{0}}\right)^{\frac{1}{2}}\left(\frac{c^{4}}{G}\right)\right]^{-1} m_{e} c^{2} \cong \frac{m_{e} c^{2}}{\left(F_{X}\right)_{0}}$

Here in this expression, 
$\left(F_{X}\right)_{0} \cong\left[\left(\frac{m_{e}}{m_{p}}\right)^{\frac{1}{4}}\left(\frac{m_{e}}{M_{0}}\right)^{\frac{1}{2}}\left(\frac{c^{4}}{G}\right)\right] \cong 59$ newton

can be considered as the (current cosmological) characteristic nuclear force magnitude. It is also noticed that,

$$
\sqrt{\frac{e^{2}}{4 \pi \varepsilon_{0}\left(F_{X}\right)_{0}}} \cong 1.97 \mathrm{fm}
$$

This is close to the beginning of the (current) strong interaction range $2 \mathrm{fm}$. If one is willing to guess that, the characteristic nuclear force magnitude operates in between

$$
\begin{aligned}
& \qquad\left(F_{X}\right)_{0} \cong\left[\left(\frac{m_{e}}{m_{p}}\right)^{\frac{1}{4}}\left(\frac{m_{e}}{M_{0}}\right)^{\frac{1}{2}}\left(\frac{c^{4}}{G}\right)\right] \cong 59 \text { newton and }\left(F_{Y}\right)_{0} \cong\left[\left(\frac{m_{e}}{M_{0}}\right)^{\frac{1}{2}}\left(\frac{c^{4}}{G}\right)\right] \cong 388 \text { newton } \\
& \text { If so } \sqrt{\frac{e^{2}}{4 \pi \varepsilon_{0}\left(F_{Y}\right)_{0}}} \cong 0.77 \mathrm{fm}
\end{aligned}
$$

Then their current geometric force magnitude is close to

$$
\left(F_{Z}\right)_{0} \cong \sqrt{\left(F_{X}\right)_{0}\left(F_{Y}\right)_{0}} \cong\left(\frac{m_{e}}{m_{p}}\right)^{\frac{1}{8}}\left[\left(\frac{m_{e}}{M_{0}}\right)^{\frac{1}{2}}\left(\frac{c^{4}}{G}\right)\right] \cong 152 \text { newton. }
$$

To a very accuracy it is noticed that,

$$
\sqrt{\frac{e^{2}}{4 \pi \varepsilon_{0} \sqrt{\left(F_{X}\right)_{0}\left(F_{Y}\right)_{0}}}} \cong \sqrt{\frac{e^{2}}{4 \pi \varepsilon_{0}\left(F_{Z}\right)_{0}}} \cong 1.23 \mathrm{fm}
$$

This is very close to the presently believed characteristic nuclear charge radius. In an unified picture, it can be expressed as

$$
\left(R_{C}\right)_{0} \cong\left[\left(\frac{m_{p}}{m_{e}}\right)^{\frac{1}{16}}\left(\frac{M_{0}}{m_{e}}\right)^{\frac{1}{4}}\right] \sqrt{\frac{e^{2} G}{4 \pi \varepsilon_{0} c^{4}}} \cong 1.23 \mathrm{fm}
$$

In unification program - this coincidence cannot be ignored and must be given a top priority in confirming the basics of unified physics.

If $M_{p}$ is the Planck mass and $R_{0} \cong\left(c / H_{0}\right)$ is the current gravitational and electromagnetic interaction range, it is noticed that,

$$
\ln \left(\frac{m_{e} R_{0}^{2}}{M_{P} R_{s}^{2}}\right) \cong 137.2 \cong \frac{1}{\alpha} .
$$

Now the fundamental question to be answered is - Is fine structure ratio - a cosmological variable? If it is possible, then the reduced Planck's constant also seems to be a cosmological variable. From relations (9) and (18) it is noticed that,

$$
\ln \left(\sqrt{\frac{m_{p}}{M_{0}}} \cdot \frac{m_{e} R_{0}^{2}}{M_{p} R_{p}^{2}}\right) \cong \frac{1}{\alpha}
$$

where $M_{p} \cong \sqrt{\frac{\hbar c}{G}}$ is the Planck mass, $R_{p} \cong \sqrt{\frac{G \hbar}{c^{3}}}$ is the Planck size and $m_{p}$ is the proton rest mass. On re-arranging, to a 
great surprise it is noticed that

$\frac{\hbar c}{G m_{p} \sqrt{M_{0} m_{e}}} \cong 0.99753$

This ratio is very close to unity! One should not ignore this strange and peculiar observation. From this relation it can be suggested that, along with the cosmic variable $H_{0}$, on the cosmological time scale, there exists one variable physical quantity in the presently believed atomic and nuclear physical constants. 'Rate of change' in its magnitude may be a measure of the present cosmic acceleration. Thus independent of the cosmic red shift and CMBR observations, from atomic and nuclear physics, cosmic acceleration can be verified. Above relation can be expressed as [46]

$\hbar \cong \sqrt{\frac{M_{0}}{m_{e}}} \cdot \frac{G m_{p} m_{e}}{c} \cong \sqrt{\frac{M_{0}}{m_{e}}} \cdot\left(\frac{G m_{p}}{c^{2}}\right) \cdot\left(m_{e} c\right) \cong 1.0572 \times 10^{-34} \mathrm{~J} . \mathrm{sec}$

Here $\left(M_{0} / m_{e}\right)$ can be considered as the number of electrons in the present universe of mass, $M_{0} \cong\left(c^{3} / 2 G H_{0}\right)$. If so, present Hubble's constant can be expressed as

$H_{0} \cong \frac{G m_{p}^{2} m_{e} c}{2 \hbar_{0}^{2}} \cong 70.743 \mathrm{~km} / \mathrm{sec} / \mathrm{Mpc}$

Another interesting relation is

$\hbar_{0}^{2} H_{0} \cong \hbar_{t}^{2} H_{t} \cong \frac{G m_{p}^{2} m_{e} c}{2} \cong$ constant

\subsection{To understand the discrete nature of revolving electrons' angular momentum}

In any bound system, 'operating force' only plays major role in maintaining the 'existence of the bound system' and 'angular momentum' is one of the result. If one is able to make the operating force as discrete, then automatically one can observe a discrete structure like discrete radii, discrete angular momentum and discrete energy levels. Alternatively if atomic nucleus constitutes any fixed number of protons and any fixed number of neutrons, it is possible to guess that, nuclear mass is discrete. If nuclear matter is discrete, it is also possible to have a discrete atomic structure. With this idea, from relation (21) revolving electron's discrete angular momentum can be expressed as

$$
n . \hbar_{0} \cong \sqrt{\frac{M_{0}}{m_{e}}} \cdot \frac{\left(n \cdot m_{p} c^{2}\right)\left(m_{e} c^{2}\right)}{\left(\frac{c^{4}}{G}\right) c}
$$

where $\left(n . m_{p} c^{2}\right)$ represents the integral proton rest energy and $\mathrm{n}=1,2,3 \ldots$ In terms of the characteristic force magnitude, above relation can be expressed as

$$
n . \hbar_{0} \cong \frac{\left(n . m_{p} c^{2}\right)\left(m_{e} c^{2}\right)}{\left(F_{Y}\right)_{0} c}
$$

From this it can be suggested that,

$$
\frac{F_{C}}{\left(F_{Y}\right)_{0}} \cong \frac{\hbar_{0} c}{G m_{e} m_{p}}
$$

It is also noticed that,

$\hbar \cong\left(\frac{2 G m_{p}}{c^{2} R_{p}}\right) \cdot\left(\frac{m_{e} c^{2}}{H_{0}}\right) \cong \frac{2 G m_{p} m_{e}}{R_{p} H_{0}} \cong\left(\frac{c}{H_{0} R_{p}}\right)\left(\frac{2 G m_{p}}{c^{2}}\right)\left(m_{e} c\right)$

where $R_{p}$ is the 'rms charge radius' of proton [45]. This is another accurate relation that connects the universe and the atom. With different experimental methods $R_{p}$ magnitude varies from 0.84184(67) fm to 0.895(18) fm. The two best quoted values of the rms radius of proton are $0.87680(690) \mathrm{fm}$ and $0.84184(67) \mathrm{fm}$. If electron revolves round the proton, this expression can be given a chance. If so, present Hubble's constant can be expressed as 
$H_{0} \cong \frac{2 G m_{p} m_{e}}{R_{p} \hbar_{0}} \cong(67.88$ to 70.69$) \mathrm{km} / \mathrm{sec} / \mathrm{Mpc}$

If $R_{p} \cong 0.84184(67) \mathrm{fm} \rightarrow \mathrm{H}_{0} \cong 70.69 \mathrm{~km} / \mathrm{sec} / \mathrm{Mpc}$ and if $R_{p} \cong 0.87680 \mathrm{fm} \rightarrow \mathrm{H}_{0} \cong 67.88 \mathrm{~km} / \mathrm{sec} / \mathrm{Mpc}$. Another interesting relation is

$\hbar_{0} H_{0} \cong \hbar_{t} H_{t} \cong \frac{2 G m_{p} m_{e}}{R_{p}} \cong$ constant

Now from relation (27)

$\hbar_{0} \cong\left(\frac{4 G M_{0}}{c^{2} R_{p}}\right) \cdot \frac{G m_{p} m_{e}}{c}$

Here in this expression, the very interesting and peculiar expression is $\left(\frac{4 G M_{0}}{c^{2} R_{p}}\right) \cong 2\left(\frac{c}{H_{0} R_{p}}\right)$. This resembles the

Einstein's famous space - time curvature relation in case of 'bending of light ray'. Now the famous uncertainty relation takes the following simple form.

$\frac{\hbar_{0}}{2} \cong\left(\frac{2 G M_{0}}{c^{2} R_{p}}\right) \cdot \frac{G m_{p} m_{e}}{c} \cong\left(\frac{R_{0}}{R_{p}}\right) \cdot \frac{G m_{p} m_{e}}{c}$

where $R_{0} \cong \frac{c}{H_{0}} \cong \frac{2 G M_{0}}{c^{2}}$. This is another interesting and peculiar observation. Its interpretation seems to be very simple but very difficult to confirm! From above relations fine structure ratio can be expressed as

$\frac{1}{\alpha_{0}} \cong\left(\frac{2 G m_{p}}{c^{2} R_{p}}\right) \cdot\left(\frac{e^{2}}{4 \pi \varepsilon_{0} m_{e} c^{2}}\right)^{-1}\left(\frac{c}{H_{0}}\right)$

Here $\left(\frac{e^{2}}{4 \pi \varepsilon_{0} m_{e} c^{2}}\right)$ is the classical radius of electron and $\left(c / H_{0}\right)$ is the assumed present gravitational and electromagnetic interaction range. From these coincidences it is possible to guess that, both $(\hbar)$ and $(\alpha)$ are compound cosmological variable quantities. But the main problem is that - variation of $(\hbar)$ seems to be linear and variation of $(1 / \alpha)$ seems to be natural logarithmic. Now the fundamental question to be answered is- How $\left(\hbar_{t}\right)$ varies with time? Whether it follows a 'natural logarithmic relation' or a 'linear relation' - to be confirmed. Answer can be obtained from analyzing the above relations. It has to be verified from past and future 'galaxy age and redshift' data analysis. Unfortunately past data is not available and we have to wait for the future data. Anyhow, current situation seems to be very ambiguous. From relations (27) and (30)

$n . \hbar_{0} \cong\left(\frac{4 G M_{0}}{c^{2} R_{p}}\right) \frac{\left(n . m_{p} c^{2}\right)\left(m_{e} c^{2}\right)}{\left(\frac{c^{4}}{G}\right) c}$

It is noticed that,

$\left(F_{Y}\right)_{0} \cong\left(\frac{4 G M_{0}}{c^{2} R_{p}}\right)^{-1}\left(\frac{c^{4}}{G}\right) \cong\left(\frac{c^{2} R_{p}}{4 G M_{0}}\right)\left(\frac{c^{4}}{G}\right) \cong\left(\frac{H_{0} R_{p}}{2 c}\right)\left(\frac{c^{4}}{G}\right) \cong 397$ newton

From above relations it is also possible to show that, 'rms' radius of proton increases with cosmic time. This is also a very surprising and unbelievable discovery. Current 'rms' radius of proton can be expressed as

$\left(R_{p}\right)_{0} \cong \frac{4 G \sqrt{M_{0} m_{e}}}{c^{2}}$

Even though it is very accurate, unfortunate thing is that, it is independent of the rest mass of proton and the reduced Planck's constant. Future may decide the issue. 


\subsection{The cosmic Fine structure ratio}

In atomic and nuclear physics, the fine-structure ratio $(\alpha)$ is a fundamental physical constant, namely the coupling constant characterizing the strength of the electromagnetic interaction. Being a dimensionless quantity, it has a constant numerical value in all systems of units. If $\rho_{0} c^{2}$ is the present cosmic critical energy density and $a T_{0}^{4}$ is the present cosmic thermal energy density, it is noticed that,

$\ln \sqrt{\frac{a T_{0}^{4}}{\rho_{0} c^{2}} \cdot \frac{4 \pi \varepsilon_{0} G M_{0}^{2}}{e^{2}}} \cong\left(\frac{1}{\alpha}\right)$

At present, if $H_{0}$ is close to $71 \mathrm{~km} / \mathrm{sec} / \mathrm{Mpc}$ and $T_{0} \cong 2.725{ }^{0} \mathrm{~K}$, obtained value of $(1 / \alpha)_{0}$ is 137.04773 . Note that, from unification point of view, till today role of dark energy or dark matter is unclear and undecided. Their laboratory or physical existence is also not yet confirmed. In this critical situation this application can be considered as a key tool in particle cosmology. Note that large dimensionless constants and compound physical constants reflect an intrinsic property of nature. Above relation takes the following form.

$\ln \sqrt{\frac{2 \pi}{3} \cdot \frac{4 \pi \varepsilon_{0}\left(a T_{0}^{4}\right) c^{4}}{e^{2} H_{0}^{4}}} \cong\left(\frac{1}{\alpha}\right)_{0}$

After simplification, it can be interpreted as follows. Total thermal energy in the present Hubble volume can be expressed as,

$\left(E_{T}\right)_{0} \cong a T_{0}^{4} \cdot \frac{4 \pi}{3}\left(\frac{c}{H_{0}}\right)^{3}$

If $\left(c / H_{0}\right)$ is the present electromagnetic interaction range, then present electromagnetic potential can be expressed as

$\left(E_{e}\right)_{0} \cong \frac{e^{2}}{4 \pi \varepsilon_{0}\left(c / H_{0}\right)}$

Now inverse of the present fine structure ratio can be expressed as

$\left(\frac{1}{\alpha}\right)_{0} \cong \ln \sqrt{\frac{\left(E_{T}\right)_{0}}{2\left(E_{e}\right)_{0}}}$

Here, in RHS, denominator ' 2 ' may be a representation of total thermal energy in half of the cosmic sphere or thermal energy of any one pole of the cosmic sphere. This is a simple and direct application of the proposed assumptions. Thus at any cosmic time,

$\left(\frac{1}{\alpha}\right)_{t} \cong \ln \sqrt{\frac{\left(E_{T}\right)_{t}}{2\left(E_{e}\right)_{t}}}$

This may be considered as the cosmological definition for the fine structure ratio. Semi empirically to a good approximation, it is noticed that,

$\frac{1}{\alpha_{t}} \cong \ln \left(\frac{x}{1+\ln (x)}\right)$

Here $x \cong \sqrt{\frac{4 \pi \varepsilon_{0} G M_{t}^{2}}{e^{2}}}$.If $M_{t} \rightarrow \sqrt{\frac{e^{2}}{4 \pi \varepsilon_{0} G}},\left(\frac{1}{\alpha}\right)_{t} \rightarrow 0$.

With this relation and with reference to the current magnitude of the fine structure ratio, obtained value of the present Hubble's constant is close to $71.75 \mathrm{~km} / \mathrm{sec} / \mathrm{Mpc}$. Now the reduced Planck's constant can be expressed as

$\hbar_{t} \cong \frac{1}{\alpha_{t}} \cdot \frac{e^{2}}{4 \pi \varepsilon_{0} c}$

From this relation it is possible to say that, cosmological rate of change in fine structure ratio, $(d \alpha / d t)$ or $(d \hbar / d t)$ may be considered as an index of the future cosmic expansion or acceleration. More recently, theoretical interest in varying constants (not just $\alpha$ ) has been motivated by string theory [47] and other such proposals for going beyond the Standard 
Model of particle physics. In October 2011 Webb et al. reported a variation in $\alpha$ dependent on both redshift and spatial direction [48]. This idea will certainly motivate anybody to think about the variation of $h$. But till today from ground based laboratory experiments no variation was noticed in the magnitude of the fine structure ratio. Future experiments and observations may reveal the real picture. Very recent observations [49] found no spatial variation in $\alpha$ even at a redshift of $10^{3}$. This is very good signal for the saturation or halting of the primordial cosmic expansion.

Including the $\mathrm{CMB}$ radiation energy density and the observed matter-energy density, in this connection, authors observed so many interesting relations in unification program. The proposed relations (9) to (43) are best examples for this. In the following sections, authors proposed important observations and concepts related to Mach's principle, Hubble volume and the four fundamental cosmological interactions.

\section{Possible assumptions in unified cosmic physics}

\subsection{About the light speed rotating black holes}

Whether a black hole is the end point of matter or it is the base point of observable cosmic matter - is still a mystery in the current physics world. There is no perfect theory that defines the lower and upper limits of a massive black hole. As the radius of a black hole is directly proportional to its mass, its density decreases as its mass increases. If mass is at lower side, density will be very high, if mass is higher side, density will be very low. If mass of black hole is $10^{40} \mathrm{~kg}$, then black hole's density will be close to $0.73 \mathrm{Kg} / \mathrm{m}^{3}$ and is less than the air density. The fundamental question to be understood is: if black hole mass is too high and density is too less, inside a black hole whether our known physical laws will work? Answer is beyond the scope of current physics.

Generally it is believed that, black holes are formed by gravitational collapse of massive stars. Gravitational collapse requires great density. In the current epoch of the universe these high densities are only found in stars, but in the early universe shortly after the big bang densities were much greater, possibly allowing for the creation of black holes. The high density alone is not enough to allow the formation of black holes since a uniform mass distribution will not allow the mass to bunch up. In order for primordial black hole to form in such a dense medium, there must be initial density perturbations that can then grow under their own gravity. Different models for the early universe vary widely in their predictions of the size of these perturbations. Various models predict the creation of black holes, ranging from a Planck mass to hundreds of thousands of solar masses. Primordial black holes could thus account for the creation of any type of black hole.

Once a black hole has been formed, it can continue to grow by absorbing additional matter. Any black hole will continually absorb gas and interstellar dust from its direct surroundings and omnipresent cosmic background radiation. This is the primary process through which super massive black holes seem to have grown. Astronomers believe that black holes that are as large as a billion solar masses can be found at the centre of most galaxies. Here the fundamental questions to be answered are: If the galactic central black hole mass is 10 billion solar masses and density is less than 1 $\mathrm{kg} / \mathrm{m}^{3}$ - with such a small density and large mass, without collapsing - how it is able to hold a gigantic galaxy? What force makes the black hole stable? Recent observations indicate that, instead of collapsing, galactic central black holes are growing faster and spinning faster than they expected. In 2013 February, using Nasa's newly launched NuStar telescope and the European Space Agency's workhorse XMM-Newton, an international team observed high-energy Xrays released by a super massive black hole in the middle of a nearby galaxy. They calculated its spin at close to the speed of light: 670million mph [50,51]. This is really a very good news for the beginning of 'Black hole cosmology' If universe constitutes so many galaxies, if each galaxy constitutes a central fast growing and (light speed) spinning black hole and if black hole geometry is more intrinsic than its 'mass' and 'mass density', then considering universe as a 'growing and light speed rotating black hole' may not be far away from reality. Unknowingly the fundamental physical laws are being developed, being executed and being proven inside and under the background of a growing and light speed rotating black hole.

\subsection{Possible assumptions}

The possible assumptions in unified cosmic physics can be expressed in the following way. Please note that, with the proposed assumptions and observations/discoveries whether we are falling in an intellectual singularity or coming out from the intellectual singularity future may decide.

A) With reference to the elementary charge and with similar to the Planck mass, a new mass unit can be constructed in the following way. It can be called as the 'Coulomb mass'. 
$M_{C} \cong \sqrt{\frac{e^{2}}{4 \pi \varepsilon_{0} G}} \cong 1.859210775 \times 10^{-9} \mathrm{Kg} \cong 1.042941 \times 10^{18} \mathrm{GeV} / \mathrm{c}^{2}$

It represents the characteristic mass of elementary charge in unification program. It can be considered as the seed of galactic matter or galactic central black hole. It can also be considered as the seed of any cosmic structure.

B) At any time Hubble length $\left(c / H_{t}\right)$ can be considered as the gravitational or electromagnetic interaction range.

C) At any time, $H_{t}$ being the angular velocity, universe can be considered as a growing and light speed rotating primordial black hole [14-17],[52-58]. Thus at any given cosmic time,

$$
R_{t} \cong \frac{2 G M_{t}}{c^{2}} \cong \frac{c}{H_{t}} \quad \text { and } \quad M_{t} \cong \frac{c^{3}}{2 G H_{t}}
$$

when $M_{t} \rightarrow M_{C}, R_{C} \cong \frac{2 G M_{C}}{c^{2}}$ and $H_{C} \cong \frac{c}{R_{C}} \cong \frac{c^{3}}{2 G M_{C}}$. can be considered as the characteristic initial measurements of the universe. Similarly

and $R_{0} \cong \frac{2 G M_{0}}{c^{2}} \cong \frac{c}{H_{0}}$ and $\mathrm{M}_{0} \cong \frac{c^{3}}{2 G H_{0}}$ can be considered as the characteristic current measurements of the universe.

D) Reduced Planck's constant or the characteristic angular momentum of the revolving electron increases with cosmic time.

E) Characteristic nuclear size increases with cosmic time. In this regard, to a great surprise, it is noticed that,

$$
\left(R_{0} R_{C}^{2}\right)^{\frac{1}{3}} \cong\left(\frac{M_{0}}{M_{C}}\right)^{\frac{1}{3}} R_{C} \cong\left(\frac{V_{0}}{V_{C}}\right)^{\frac{1}{9}} \frac{2 G M_{C}}{c^{2}} \cong 9.97 \times 10^{-16} \mathrm{~m} \approx \text { One fermi }
$$

Here, $V_{0} \cong \frac{4 \pi}{3} R_{0}^{3}$ and $V_{C} \cong \frac{4 \pi}{3} R_{C}^{3}$. Please note that, 'mass' is a 3 dimensional property and 'radius' is a one dimensional property. So logically the ratio $\left(\frac{M_{0}}{M_{C}}\right)^{\frac{1}{3}}$ can be considered as a cosmological proportionality ratio in estimating any 'characteristic sphere' radius. As a cosmologist or nuclear physicist, one must make a serious attempt to understand this strange coincidence. This idea can be considered as a key tool in judging this paper.

\section{To understand the cosmic redshift}

During cosmic evolution, as cosmic time increases, hydrogen atom emit photons with increased quanta of energy. Thus past light quanta emitted from old galaxy will have less energy and show a red shift with reference to our galaxy. During journey light quanta will not lose energy and there will be no change in light wavelength. If one is willing to think in this new direction, it may not a big problem to invoke mechanisms for this new concept. At present if we represent $\hbar \cong \hbar_{0}$, in the past the operating reduced Planck's constant was $\hbar_{t}$ and its magnitude was less than $\hbar_{0}$. Thus at any given cosmic time $t, \frac{d(\hbar)}{d t}$ can be considered as a measure of cosmic rate of expansion. As time is passing, one can expect a very small change in $\frac{d(\hbar)}{d t}$ and it may be beyond the scope of experimental accuracy. But to have a rapid (detectable) change in $\frac{d(\hbar)}{d t}$ present cosmic time should run fast or should accelerate. Different mechanisms can be invoked $[14,15]$ and it is possible to show that, potential energy of electron in hydrogen atom is directly proportional to $\hbar^{2}$. Bohr's second postulate which suggests that potential energy of electron in hydrogen atom is inversely proportional to $\hbar^{2}$ seems to be a coincidence. It may be noted that, increased redshifts and increased distances forced Edwin Hubble to propose the Hubble's law. If it is possible to show that, (from the observer) older galaxy's distance increases with its 'age', then automatically the concepts 'galaxy receding' and 'accelerating universe' can be put for a revision at fundamental level. With reference to our laboratory or our galaxy, the basic or original definition of present/current redshift $\left(z_{0}\right)$ seems to be: 
$z_{0} \cong \frac{E_{0}-E_{G}}{E_{0}} \cong \frac{\lambda_{G}-\lambda_{0}}{\lambda_{G}} \leq 1$

But not $z_{0} \cong \frac{E_{0}-E_{G}}{E_{G}} \cong \frac{\lambda_{G}-\lambda_{0}}{\lambda_{0}}$.

Here $E_{0} \cong \frac{h c}{\lambda_{0}}$ is the energy of photon at our galaxy/laboratory and $E_{G} \cong \frac{h c}{\lambda_{G}}$ is the energy of photon at the observed galaxy when it was emitted. Similarly $\lambda_{G}$ is the wave length of light received from observed galaxy and $\lambda_{0}$ is the wave length of light in laboratory. Even though both relations are ad-hoc and not absolute definitions, compared to relation (48), relation (47) seems to be some what reliable. Very interesting thing is that, when redshift is very small (up to $z \approx 0.01$ ), both relations almost all will give the same result. Important point to be noticed is that, by Hubble's time the maximum redshift noticed was 0.003 and was less than 0.01 . One should not ignore this fact. Note that, based on the increasing value of the Planck's constant, present red shift $\left(z_{0}\right)$ will be directly proportional to age difference between our galaxy and observed galaxy or time taken by light to reach our galaxy from the old galaxy $(\Delta t)$. Thus $z_{0} \propto \Delta t$ and

$z_{0} \cong H_{0} \Delta t$

Here $H_{0}$ is the proportionality constant. In this way $H_{0}$ can be incorporated directly. Time taken by light to reach our galaxy or the age difference of our galaxy and observed galaxy can be expressed as,

$\Delta t \cong \frac{z_{0}}{H_{0}}$.

$c \Delta t \cong z_{0} \cdot \frac{c}{H_{0}}$.

To confirm this, absolute methods (that are free from redshift) for estimating galaxy age can be considered. Then the basic and original definition of 'galaxy receding' and 'accelerating universe' concepts can be eliminated and a 'decelerating or expanded universe' concept can be continued without any difficulty. Now the fundamental question to be answered is: If $\left(\hbar_{t}\right)$ takes the role of $(\hbar)$, how to define the red shift? Considering $\left(\frac{E_{0}-E_{G}}{E_{0}}\right)$ authors proposed a simple solution to this problem. With different galaxies and with different $(\Delta t)$,

$H_{0} \cong\left(\frac{z_{0}}{\Delta t}\right)_{G_{1}} \cong\left(\frac{z_{0}}{\Delta t}\right)_{G_{2}} \cong\left(\frac{z_{0}}{\Delta t}\right)_{G_{3}}$

where $G_{1}, G_{2}$ and $G_{3}, .$. represent different galaxies. In the past, photon energy can be expressed as

$\left(E_{\text {pho }}\right)_{t} \cong \frac{2 \pi \hbar_{t} c}{\lambda_{G}}$

Current photon energy can be expressed as

$\left(E_{\text {pho }}\right)_{o} \cong \frac{2 \pi \hbar_{0} c}{\lambda_{0}}$

Thus for any galaxy, where $\hbar_{t}$ was playing a key role, corresponding present cosmic red shift can be expressed as

$z_{0} \cong \frac{\left(E_{P h o}\right)_{0}-\left(E_{P h o}\right)_{G}}{\left(E_{P h o}\right)_{0}} \cong 1-\left[\left(\frac{\hbar_{t}}{\hbar_{0}}\right)\left(\frac{\lambda_{0}}{\lambda_{G}}\right)\right]$

Whatever may be the expression, including the relations (47) and (48), various definitions of red shift seem to be ad-hoc and not absolute $[59,60]$. Hence with redshift concept - one may not be able to understand the actual rate of cosmic expansion. From cosmology point of view, the real picture of cosmic rate of expansion can be understood from the 'rate of decreasing' cosmic thermal energy density. 


\section{Applications of the proposed assumptions}

\subsection{The cosmic matter density}

Approximate relation between cosmic volume density $\left(\rho_{v}\right)_{t}$ and matter density $\left(\rho_{m}\right)_{t}$ can be expressed as

$\left(\rho_{m}\right)_{t} \cong\left[1+\ln \sqrt{\frac{4 \pi \varepsilon_{0} G M_{t}^{2}}{e^{2}}}\right]^{-1}\left(\frac{3 H_{t}^{2}}{8 \pi G}\right)$

If $M_{t} \rightarrow \sqrt{\frac{e^{2}}{4 \pi \epsilon_{0} G}},\left(\rho_{m}\right)_{C} \rightarrow \frac{3 H_{C}^{2}}{8 \pi G}$. Instead of the 'Planck mass', initial conditions can be addressed with $M_{C} \cong \sqrt{\frac{e^{2}}{4 \pi \varepsilon_{0} G}}$. Note that, at present obtained matter density can be compared with the elliptical and spiral galaxy matter density. Based on the average mass-to-light ratio for any galaxy [61,62]

$\left(\rho_{m}\right)_{0} \cong 1.5 \times 10^{-32} \eta h_{0}$ gram $/ \mathrm{cm}^{3}$

where for any galaxy, $\langle\mathrm{M} / \mathrm{L}\rangle$ Galaxy $=\eta\langle\mathrm{M} / \mathrm{L}\rangle$ Sun and the number: $h_{0} \cong \frac{H_{0}}{100 \mathrm{Km} / \mathrm{sec} / \mathrm{Mpc}} \cong \frac{71.0}{100} \cong 0.71$. Note that elliptical galaxies probably comprise about $60 \%$ of the galaxies in the universe and spiral galaxies are thought to make up about $20 \%$ of the galaxies in the universe. Almost $80 \%$ of the galaxies are in the form of elliptical and spiral galaxies. For spiral galaxies, $\eta h_{0}^{-1} \cong 9 \pm 1$ and for elliptical galaxies, $\eta h_{0}^{-1} \cong 10 \pm 2$. For our galaxy inner part, $\eta h_{0}^{-1} \cong \cong 6 \pm 2$. Thus the average $\eta h_{0}^{-1}$ is very close to 8 to 9 and its corresponding matter density is $(6.05$ to 6.8$) \times 10^{-32} \mathrm{gram} / \mathrm{cm}^{3}$.

\subsection{The cosmic thermal energy density}

At any given cosmic time, ratio of cosmic volume energy density and cosmic thermal energy density can be expressed as

$$
\left(\frac{\rho_{v} c^{2}}{a T^{4}}\right)_{t} \cong\left[1+\ln \sqrt{\frac{4 \pi \varepsilon_{0} G M_{t}^{2}}{e^{2}}}\right]^{2}
$$

This is an observation and can be considered as a discovery. If so, at any given cosmic time, thermal energy density can be expressed as

$$
a T_{t}^{4} \cong\left[1+\ln \left(\frac{M_{t}}{M_{C}}\right)\right]^{-2}\left(\frac{3 H_{t}^{2} c^{2}}{8 \pi G}\right)
$$

If $M_{t} \rightarrow M_{C}, a T_{t}^{4} \rightarrow \frac{3 H_{C}^{2} c^{2}}{8 \pi G} \cong a T_{C}^{4}$ and can be considered as the characteristic initial thermal energy density of the universe. Please note that at present

$$
a T_{0}^{4} \cong\left[1+\ln \left(\frac{M_{0}}{M_{C}}\right)\right]^{-2}\left(\frac{3 H_{0}^{2} c^{2}}{8 \pi G}\right)
$$

If present $H_{0}$ is close to $71.1 \mathrm{~km} / \mathrm{sec} / \mathrm{Mpc}$, obtained CMBR temperature is $2.725{ }^{0} \mathrm{~K}$. Note that from above relations (943), value of $H_{0}$ can be obtained and can be implemented in relation (60). If one is willing to think in this new direction, certainly other new relations can also be obtained. At any given cosmic time, without considering the quantum theory of light, equivalent CMBR thermal energy density can be obtained in this way. Really this is a miracle. Its interpretation seems to be interesting. Compared to the complicated redshift observations, this proposal seems to be simple and reliable. 


\section{Discussion \& Conclusions}

If universe constitutes so many galaxies, if each galaxy constitutes a central fast growing and (light speed) spinning black hole and if black hole geometry is more intrinsic than its 'mass' and 'mass density', then considering universe as a 'growing and light speed rotating black hole' may not be far away from reality [19-33], [14-17], [50,51], [52-58]. Unknowingly the fundamental physical laws are being developed, being executed and being proven inside and under the background of a growing and light speed rotating black hole universe. By increasing the number of applications of Hubble mass and Hubble volume in other areas of fundamental physics, slowly and gradually and in a progressive way concepts of Black hole Cosmology can be strengthened and can also be confirmed.

With the proposed concepts and with the advancing science and technology, from the ground based laboratory experiments, from time to time the concept $d(\alpha) / d t$ or $d(\hbar) / d t$ can be put for experimental tests. As time is passing, one can expect a very small change in $d(\alpha) / d t$ or $d(\hbar) / d t$ and it may be beyond the scope of experimental accuracy. But to have a rapid (detectable) change in $d(\alpha) / d t$ or $d(\hbar) / d t$ present cosmic time should run fast or should accelerate. Whatever may be the expression, definitions of red shift seem to be ad-hoc and not absolute. Hence with redshift concept - one may not be able to understand the actual rate of cosmic expansion. From cosmology point of view, the real picture of cosmic rate of expansion can be understood from the 'rate of decreasing' cosmic thermal energy density. Well established experiments are already available by which the fine structure ratio can be estimated. Moreover, conducting an experiment in this direction is also very simple. Only thing is that the same experiment has to be repeated for several times or continuously. Thus in the near future one can expect the real picture. In this way gradually the cosmic geometry can be understood. Many experiments are under progress to detect and confirm the existence of dark matter and dark energy. Parallel to the existing modern mathematical concepts like 'higher dimensions' and 'dark energy', if one is willing to consider the proposed new concepts, definitely a unified model of cosmology and quantum physics can be developed. But it may take some time to understand and follow them.

Alternatively in a theoretical way, the proposed applications or semi empirical relations can be given a chance and the subject of elementary particle physics and cosmology can be studied in a unified manner. It is true that the proposed relations are speculative and peculiar also. By using the proposed relations and applying them in fundamental physics, in due course their role or existence can be verified. With these relations, Hubble constant can be estimated from atomic and nuclear physical constants. If one is able to derive them with a suitable mathematical model, independent of the cosmic redshift and CMBR observations, the future cosmic acceleration can be verified from atomic and nuclear physical constants. Now the key leftover things are 'nucleosynthesis' and 'structure formation'. The most important point to be noted here is that, synthesis of elementary physical constants seem to be more important and intrinsic than the 'cosmological nucleosynthesis'. Authors are working on this and will be discussed in detail in near future.

\section{Acknowledgements}

The first author is indebted to professor K. V. Krishna Murthy, Chairman, Institute of Scientific Research on Vedas (ISERVE), Hyderabad, India and Shri K. V. R. S. Murthy, former scientist IICT (CSIR) Govt. of India, Director, Research and Development, I-SERVE, for their valuable guidance and great support in developing this subject.

\section{References}

[1] Saul Perlmutter, Supernovae, Dark Energy and the Accelerating Universe, American Institute of Physics, Physics Today, pp53-60, April 2003.

[2] Hubble E. P, A relation between distance and radial velocity among extra-galactic nebulae, PNAS, 1929, vol. 15, 1929, pp.168-173.

[3] Hubble, E.P, The 200-inch telescope and some problems it may solve. PASP, 59, pp153-167, 1947.

[4] Bruno Leibundgut and Jesper Sollerman . A cosmological surprise: the universe accelerates. Europhysics News (2001) Vol. 32 No. 4

[5] P. J. E. Peebles and Bharat Ratra (2003). The cosmological constant and dark energy. Reviews of Modern Physics 75 (2): $559-606$

[6] Joshua Frieman, Michael Turner and Dragan Huterer, Dark Energy and the Accelerating Universe. Ann. Rev. Astron. Astrophys.46: pp385432, 2008.

[7] Mustapha Ishak, Remarks on the Formulation of the Cosmological Constant/Dark Energy Problems, Found Phys, 37,pp1470-1498, 2007.

[8] J. W. Moffat, Modified Gravity Or Dark Matter? Online Available: http://arxiv.org/abs/1101.1935v2

[9] Narlikar, J.V, Vishwakarma,R.G. and Burbidge.G., Interpretations of the Accelerating Universe, The Publications of the Astronomical Society of the Pacific, Volume 114, Issue 800, pp. 1092-1096, 2002.

[10] R. G. Vishwakarma and J. V. Narlikar, Modeling repulsive gravity with creation, J. Astrophys. Astr. vol- 28, pp17-27, 2007.

[11] Arman Shafielooa, Varun Sahnib and Alexei A. Starobinsky, Is cosmic acceleration slowing down? Phys. Rev. D 80, pp101301, 2009.

[12] Peter Rowlands. Problems on the standard model of cosmology and the solutions arising from Santillie's theory. Hadronic Journal. Vol-35, No-6, 593-608, 2012. 
[13] Ruggero Maria Santilli . Experimental Verifications of Isoredshift with Possible Absence of Universe Expansion, Big Bang, Dark Matter, and Dark Energy. The Open Astronomy Journal, 2010, 3, 126-144.

[14] U. V. S. Seshavatharam, S. Lakshminarayana, B.V.S.T. Sai. Is red shift - an index of galactic 'atomic light emission' mechanism? International Journal of Physics, 2013, Vol. 1, No. 3, 49-64

[15] U. V. S. Seshavatharam, S. Lakshminarayana, B.V.S.T. Sai. . Nucleus, Atom and the Universe - a combined study. International Journal of Advanced Astronomy, 1 (1) (2013) 1-12

[16] U.V.S. Seshavatharam, Physics of Rotating and Expanding Black Hole Universe, Progress in Physics, 2: 7-14, 2010.

[17] U.V.S. Seshavatharam. The Primordial Cosmic Black Hole and the Cosmic Axis of Evil. International Journal of Astronomy, 1(2): 20-37, 2012.

[18] Hawking S.W. A Brief History of Time. Book. Bantam Dell Publishing Group. 1988

[19] Michael J. Longo, Detection of a Dipole in the Handedness of Spiral Galaxies with Redshifts z 0.04, Phys. Lett. B 699, 224-229 .2011

[20] S.W. Hawking. On the rotation of the universe. Mon. Not. Royal. Astr. Soc. 142, 129-141. 1969.

[21] C Sivaram and Kenath Arun, Primordial Rotation of the Universe, Hydrodynamics, Vortices and Angular Momenta of Celestial Objects. The Open Astronomy Journal, 2012, 5, 7-11

[22] S.-C. Su and M.-C. Chu. Is the universe rotating? Astrophysical Journal, 703 354. doi:10.1088/0004-637X/703/1/354.

[23] Sidharth,B.G.. Is the Universe Rotating? Prespacetime Journal. October 2010, Vol. 1, Issue 7, pp. 1168-1173.

[24] E. Kajari et al. Rotation in relativity and the propagation of light. Proceedings of the International School of Physics "Enrico Fermi", Course CLXVIII, pp. 45-148 (2009)

[25] Istvan Nemeti et al. Visualizing ideas about Godel-type rotating universes. Godel-type Spacetimes: History and New Developments.www.math-inst.hu/pub/algebraic-logic/godunivisu-revised.pdf (2009)

[26] Marcelo Samuel Berman. A General Relativistic Rotating Evolutionary Universe. Astrophys. Space Sci.314:319-321,2008

[27] G. Chapline et al. Tommy Gold Revisited: Why Does Not The Universe Rotate? AIP Conf.Proc.822:160-165, 2006. http://arxiv.org/abs/astro$\mathrm{ph} / 0509230$

[28] Robert V Gentry. New Cosmic Center Universe Model Matches Eight of Big Bang's Major Predictions Without The F-L Paradigm. CERN preprint, EXT-2003-022, 14 Apr 2003.

[29] Kurt Godel. Rotating Universes in General Relativity Theory. Proceedings of the international Congress of Mathematicians in Cambridge, 1: $175-81,1950$.

[30] Dmitri Rabounski. On the Speed of Rotation of Isotropic Space: Insight into the Redshift Problem. The Abraham Zelmanov Journal, Vol. 2, 2009, 208-223.

[31] Mauro Dorato. On becoming cosmic time and rotating universes. Time, Reality and Experience (provisional title), Royal Institute of Philosophy Series, Cambridge University Press, 2001.

[32] Yuri N. Obukhov. On physical foundations and observational effects of cosmic Rotation. Published in Colloquium on Cosmic Rotation, Eds M. Scherfner, T. Chrobok and M. Shefaat (Wissenschaft und Technik Verlag: Berlin, 2000) pp. 23-96. http://arxiv.org/abs/astroph/0008106v1,7August 2000.

[33] Barrow JD, Juszkiewicz R, Sonoda DH. Universal rotation - How large can it be? Mon Not R Astron Soc 1985; 213 : 917.

[34] Narlikar J.V. Direct Particle Formulation of Mach's Principle. Einstein Studies, vol. 6: Mach's Principle: From Newton's Bucket to Quantum Gravity, pp. 250-261, 1995 Birkhauser Boston, Inc. Printed in the United States

[35] P. A. M. Dirac. The cosmological constants. Nature, 139, 323, 1937.

[36] P. A. M. Dirac. A new basis for cosmology. Proc. Roy. Soc. A 165, 199, 1938

[37] Recami E. Elementary Particles as Micro-Universes, and "Strong Black-holes": A Bi-Scale Approach to Gravitational and Strong Interactions Preprint NSF-ITP-02-94. posted in the arXives as the e-print physics/0505149, and references therein.

[38] C. L. Bennett et al, Nine-Year Wilkinson Microwave Anisotropy Probe (WMAP) Observations: Final Maps and Results. Submitted to Astrophysical Journal Supplement Series. http://arxiv.org/abs/1212.5225v1.

[39] J. Huchara. Estimates of the Hubble Constant, 2010. Harvard-Smithsonian Center for Astrophysics. http://hubble.plot.dat

[40] W. L. Freedman et al. Final Results from the Hubble Space Telescope Key Project to Measure the Hubble Constant. The Astrophysical Journal 553 (1): 47-72. 2001.

[41] J. Beringer et al. Particle Data Group. Phys. Rev. D86, 010001 (2012)

[42] P. J. Mohr and B.N. Taylor, CODATA Recommended Values of the Fundamental Physical Constants.2007. http://physics.nist.gov/constants.

[43] Geiger H and Marsden E. On a diffuse reaction of the particles. Proc. Roy. Soc., Ser. A 82: 495-500, 1909.

[44] H. Yukawa. On the Interaction of Elementary Particles. Proc. Phys. Math. Soc. Jap. 17 (48). 1935

[45] Michael O. Distler et al. The RMS Charge Radius of the Proton and Zemach Moments. Phys. Lett.B. 696: 343-347,2011

[46] U. V. S. Seshavatharam and S. Lakshminarayana. Is Planck's constant - a cosmological variable? International Journal of Astronomy 2013, 2(1): 11-15 DOI: 10.5923/j.astronomy.20130201.02.

[47] Schwarz, John H.. Introduction to Superstring Theory. arXiv:hep-ex/0008017. Lectures presented at the St. Croix NATO Advanced Study Institute on Techniques and Concepts of High Energy Physics.

[48] J.K. Webb et al. Indications of a spatial variation of the fine structure constant. Physical Review letters, 107 (19) 2011

[49] Jon O'Bryan et al. Constraints on Spatial Variations in the Fine-Structure constant from Planck. http://arxiv.org/pdf/1306.1232v1.pdf

[50] Christopher S. Reynolds. Astrophysics: Black holes in a spin. Nature. 494, 432-433 (28 February 2013) doi:10.1038/494432a

[51] U. V. S. Seshavatharam. Light speed rotating black holes: The special holes. International Journal of Advanced Astronomy. 1 (1) (2013) 13-20

[52] Pathria, R. K. The Universe as a Black Hole. Nature 240 (5379):298-299. 1972 doi:10.1038/240298a0.

[53] Good, I. J. Chinese universes. Physics Today 25 (7): 15. July 1972. doi:10.1063/1.3070923.

[54] Poplawski, N. J. Radial motion into an Einstein-Rosen bridge. Physics Letters B 687 (2-3): 110-113. 2010.

[55] Zhang, Tianxi. A New Cosmological Model: Black Hole Universe. Progress in Physics, 3: 3-11, 2009

[56] Zhang, Tianxi. Cosmic microwave background radiation of black hole universe. Astrophysics and Space Science, Volume 330, Issue 1, pp 157-165. 2010.

[57] Joel Smoller and Blake Temple. Shock-wave cosmology inside a black hole. Proc Natl Acad Sci U S A. September 30; 100(20): 1121611218. 2003.

[58] Chul-Moon Yoo et al. Black Hole Universe. Time evolution. arXiv:1306.1389v1 [gr-qc] 6 Jun 2013

[59] Louis Marmet. On the Interpretation of Red-Shifts: A Quantitative Comparison of Red-Shift Mechanisms. www.marmet.org/louis/index.html.

[60] Marmet. A New Non-Doppler Redshift. Physics Essays, 1(1):24-32, 1988. Online

[61] J.V. Narlikar, Introduction to cosmology, Cambridge Univ. Press, 2002, 393- 400.

[62] Craig J. Copi et al. Big bang nucleosynthesis and the baryon density of the universe. Science 267, 192-199, 1995. http://arxiv.org/abs/astroph/9407006v2. 